\title{
The SWISS-PROT protein sequence data bank
}

\author{
Amos Bairoch and Brigitte Boeckmann ${ }^{1}$ \\ Department of Medical Biochemistry, University of Geneva, 1 rue Michel Servet, 1211 Geneva 4, \\ Switzerland and ${ }^{1}$ European Molecular Biology Laboratory, Heidelberg, FRG
}

\section{Background}

SWISS-PROT is an annotated protein sequence database established in 1986 and maintained collaboratively, since 1988, by the Department of Medical Biochemistry of the University of Geneva and the EMBL Data Library [1].

\section{Sources of the sequence data}

Sequence data in SWISS-PROT originates from three different sources:

From the Protein Sequence Database of the Protein Identification Resource (PIR) [2]

From translation of entries from the EMBL Nucleotide Sequence Database [1]

From the literature

\section{Format}

The SWISS-PROT protein sequence data bank is composed of sequence entries. Each sequence entry is composed of lines. Different types of lines, each with their own format, are used to record the various data which make up the entry. For standardization purposes the format of SWISS-PROT follows as closely as possible that of the EMBL Nucleotide Sequence Database [3]. A sample SWISS-PROT entry is shown in Figure 1.

What distinguishes SWISS-PROT from other protein sequence databases?

\section{Annotation}

To be useful to the majority of users a protein sequence database should contain as much data as possible on each of the proteins that it describes. In SWISS-PROT, as in most other sequence databases, two classes of data can be distinguished: the core data and the annotation. For each sequence entry the core data consist of the following items:

Sequence data

Citation information (bibliographical references)

Taxonomic data (description of biological source of the protein)

The annotation consists of the description of the following items:

Function(s) of the protein

Post-translational modification(s). For example carbohydrates, phosphorylation, acetylation, GPI-anchor, etc.

Domains and sites. For example calcium binding regions, ATPbinding sites, zinc fingers, homeobox, kringle, etc.

Quaternary structure

Similarities to other proteins

Disease(s) associated with deficiencie(s) in the protein

Sequence conflicts, variants, etc.
We try to include as much annotation information as possible in SWISS-PROT. To obtain this information we use, in addition to the publications that reports new sequence data, review articles to periodically update the annotations of families or groups of proteins. We also make use of external experts who send us their comments and updates concerning specific groups of proteins about which they are knowledgeable. We believe that our having systematic recourse both to publications other than those reporting the core data and to subject referees represents a unique and beneficial feature of SWISS-PROT.

In SWISS-PROT, annotation is mainly found in the comment lines (CC), in the feature table (FT) and in the keyword lines (KW). Most comments are classified by 'topics'; this approach permits the easy retrieval of specific categories of data from the database.

\section{Minimal redundancy}

We try as much as possible to minimize the redundancy of the database. Many sequence databases contain, for a given protein sequence, separate entries which correspond to different literature reports. Typically one finds a report that corresponds to a fragment of the protein sequenced at the level of the polypeptide, one or more reports reflecting the results of laboratories that have sequenced that protein at the cDNA level, and finally reports from data provided by genomic sequencing. This state of affairs has many drawbacks; for example it is not easy to obtain an overall view of the current state of the knowledge about a given protein, and similarity search programs will pick up the same protein many times during searches. In SWISS-PROT we try as much as possible to merge all these data and, if conflicts exist between various sequencing reports, we indicate them in the feature table.

\section{Integration with other databases}

It is important to provide the users of biomolecular databases with a degree of integration between the three types of sequencerelated databases (nucleic acid sequences, protein sequences and protein tertiary structures) as well as with specialized data collections. So as to provide tools that will allow software developers to implement such an integrated approach we have cross-referenced SWISS-PROT with many other databases. Currently cross-references are provided for the following databases:

EMBL Nucleotide Sequence Database [1]

PDB, the Brookhaven Protein Data Bank [4] which stores crystallographic coordinates of proteins

PIR, the protein sequence database of the Protein Identification Resource [2] 
HIV, the human retroviruses and AIDS Database [5]

OMIM, the on-line version of the book 'Mendelian Inheritance in Man' [6]

PROSITE, the Dictionary of Protein Sites and Patterns [7]

REBASE, the data base of type 2 restriction enzymes [8]

Cross-references are provided in the form of pointers to information related to SWISS-PROT entries and found in data collections other than SWISS-PROT. They are implemented using a specific type of line, the 'DR' (for Data bank Reference) line. For example the sample sequence shown in Figure 1 contains DR lines that point to EMBL, PIR, PDB, OMIM, and PROSITE. In that particular example it is therefore possible to retrieve the nucleic acid sequence(s) that encodes for that protein (EMBL), the X-ray crystallographic atomic coordinates (PDB), or the description of genetic disease(s) associated with that protein (OMIM).

\section{Content of the current release}

Release 17.0 of SWISS-PROT (February 1991) contains 20024 sequence entries, comprising $6^{\prime} 524^{\prime} 504$ amino acids abstracted from 19591 references. The data file (sequence and annotations) requires $32 \mathrm{Mb}$ of disk storage space. The database is distributed with 14 documentation and index files (user's manual, release notes, list of organisms, citation index, keyword index, etc.) that require about $9 \mathrm{Mb}$ of disk space.

\section{Distribution}

SWISS-PROT is distributed on magnetic tape and on CD-ROM by the EMBL Data Library. The CD-ROM contains both SWISSPROT and the EMBL Nucleotide Sequence Database as well as other data collections and some database query and retrieval software for MS-DOS PC compatible computers. For all enquiries regarding the subscription and distribution of SWISSPROT one should contact:

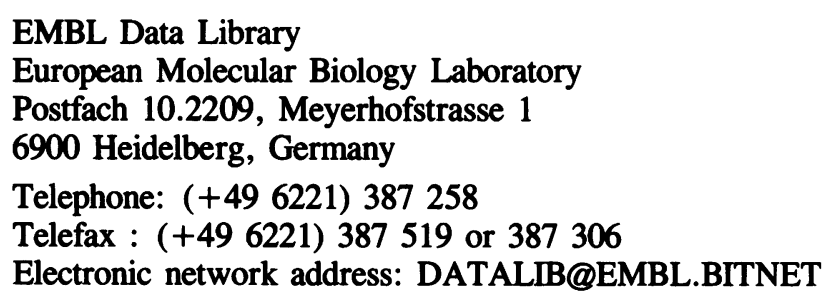

Individual sequence entries can be obtained from the EMBL File Server [9]. Detailed instructions on how to make best use of this service, and in particular on how to obtain protein sequences, can be obtained by sending to the network address NETSERV@EMBL.BITNET the following message:

HELP

HELP PROT

If you have access to a computer system linked to the Internet you can obtain SWISS-PROT using FTP (File Transfer Protocol), from the following file servers:

GenBank On-line Service [10]

Internet address: genbank.bio.net (134.172.1.160)

NCBI

Internet address: ncbi.nlm.nih.gov (130.14.20.1)

The present distribution frequency is four releases per year. No restrictions are placed on use or redistribution of the data.

\section{REFERENCES}

1. Stoehr P.J., Cameron G.N. Nucleic Acids Res. 19:2227-2230(1991)

2. Sidman K.E., George D.G., Barker W.C., Hunt L.T. Nucleic Acids Res. 16:1869-1871(1988).

3. EMBL Data Library Nucleotide Sequence Database User Manual, Release 25 of November 1990.

4. Abola E.E., Bernstein F.C., Koetzle T.F. Computational molecular biology. Sources and methods for sequence analysis, Lesk A.M., Editor, pp69-81, Oxford University Press, Oxford, (1988).

5. Human Retroviruses and AIDS 1990. A compilation and analysis of nucleic acid and amino acid sequences. Myers G., Rabson A.B. , Josephs S.F. Smith T.F. , Berzofsky J.A., Wong-Staal F., Editors. Theoretical Biology and Biophysics Group T-10, Los Alamos National Laboratory (1990).

6. McKusick V.A. Mendelian Inheritance in Man. Catalogs of autosomal dominant, autosomal recessive, and X-linked phenotypes; Ninth edition; Johns Hopkins University Press, Baltimore, (1990).

7. Bairoch A. Nucleic Acids Res. 19:2241-2245(1991).

8. Roberts R. Nucleic Acids Res. 18:2331-2366(1990).

9. Stoehr P.J., Omond R.A. Nucleic Acids Res. 17:6763-6764(1989).

10. Benton D. Nucleic Acids Res. 18:1517-1520(1990). 


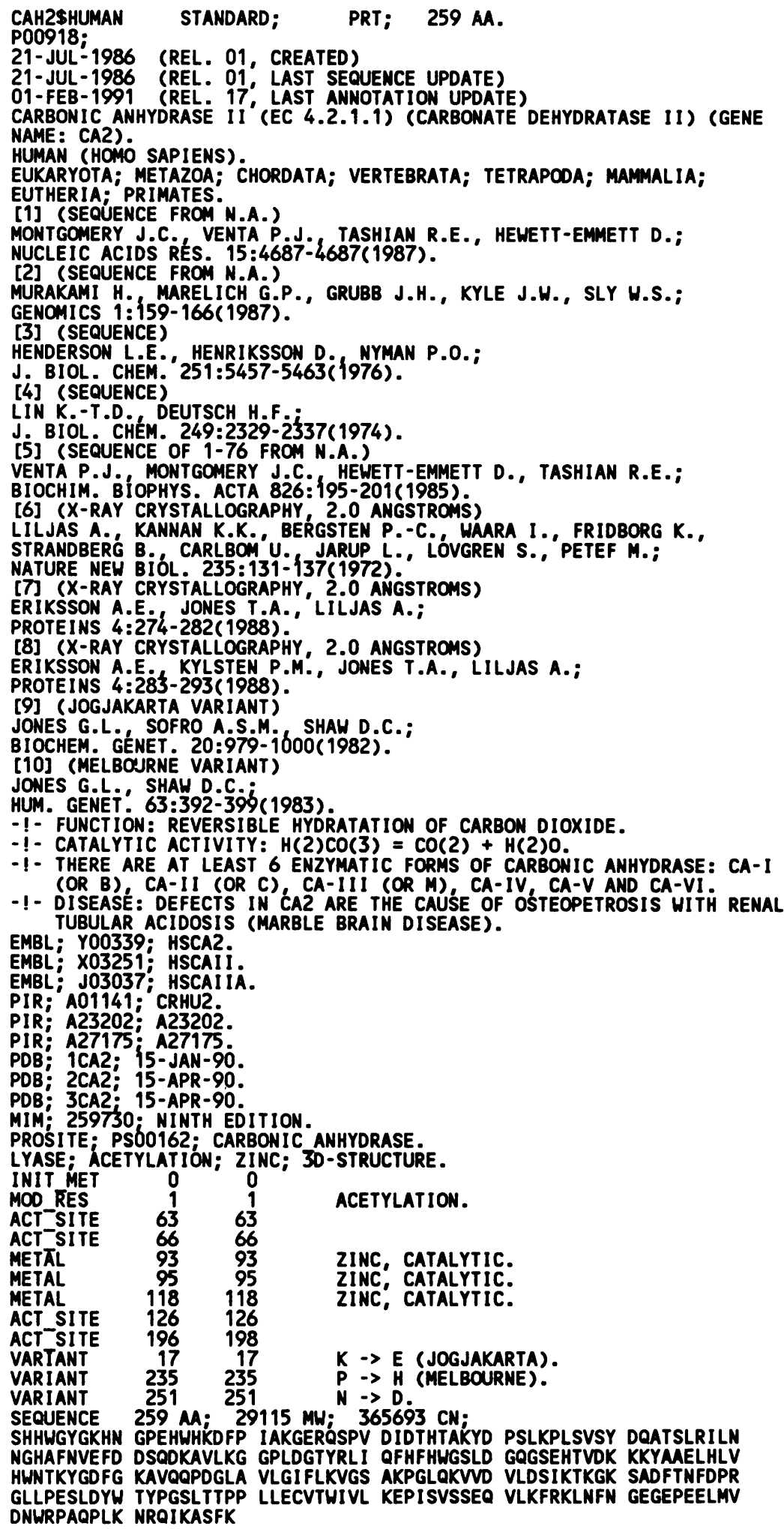

Figure 1. A sample entry from SWISS-PROT 